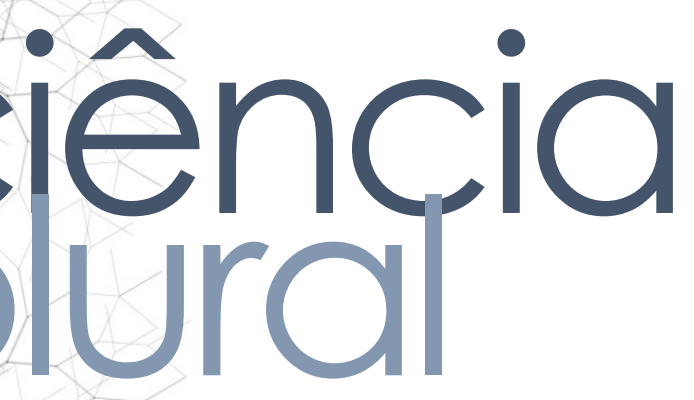

\title{
ASSISTÊNCIA À SAÚDE NA ATENÇÃO PRIMÁRIA AOS PORTADORES DE HIPERTENSÃO ARTERIAL SISTÊMICA E DIABETES MELLITUS
}

Health care in Primary Care for patients with Systemic Arterial Hypertension and Diabetes Mellitus

Atención médica en Atención Primaria para pacientes con Hipertensión Arterial Sistémica y Diabetes Mellitus

Monique da Silva Lopes • Enfermeira • Mestre em Saúde Coletiva pela Universidade Federal do Rio Grande do Norte • E-mail: moniqueslopes@msn.com

Dayane Caroliny Pereira Justino • Enfermeira • Mestre em Saúde Coletiva pela Universidade Federal do Rio Grande do Norte • E-mail: daycaroliny@hotmail.com

Fábia Barbosa de Andrade • Enfermeira • Doutora em Ciências da Saúde • Professora da Universidade Federal do Rio Grande do Norte • E-mail: fabiabarbosabr@gmail.com

Autor responsável pela correspondência:

Dayane Caroliny Pereira Justino • E-mail: daycaroliny@hotmail.com 


\section{ciêncíncia
puúl}

\section{RESUMO}

Introdução: As doenças são responsáveis por maior parte das hospitalizações e mortes no mundo. Porém, a maioria delas podem ser prevenidas por ações realizadas na Atenção Primária. Objetivo: comparar a morbidade e mortalidade por Doenças Crônicas Não Transmissíveis, Hipertensão Arterial e Diabetes Mellitus nos anos 20122014 com a cobertura da Atenção Primária na região Nordeste do Brasil. Metodologia: Trata-se de estudo ecológico, retrospectivo, realizado no nordeste brasileiro com dados dos anos de 2012 e 2014 disponíveis no Departamento de Informática do Sistema Único de Saúde. Foi realizada uma análise descritiva e analítica e correlacionada as taxas de internação e óbitos dos agravos estudados com cobertura da Atenção Primária. Resultados: Ao comparar a ocorrência de internação e óbito entre os anos de 2012 e 2014 observou-se redução no nordeste. Todavia, quando correlacionado com a cobertura da Atenção Primária, quanto maior a cobertura, maior foi a ocorrência dos agravos. Conclusões: $\mathrm{O}$ estudo revelou a necessidade de reflexão e tomada de atitude pelos gestores e profissionais de saúde considerando a continuidade do cuidado ao usuário da Atenção Primária, bem como todos os investimentos financeiros realizados pelo Ministério da Saúde junto aos municípios do Nordeste do Brasil.

Palavras-Chave: Atenção Primária à Saúde; Hipertensão; Diabetes Mellitus.

\section{ABSTRACT}

Introduction: Diseases account for most hospitalizations and deaths in the world. However, most of them can be prevented by actions taken in Primary Care. Objective: To compare morbidity and mortality due to Chronic Noncommunicable Diseases, Hypertension and Diabetes Mellitus in 2012-2014 with Primary Care coverage in northeastern Brazil. Methodology: It is an ecological and retrospective study conducted in northeastern Brazil with data from 2012 and 2014 with data available from the Department of Informatics of the Unified Health System. A descriptive and analytical analysis was conducted that correlated the rates of hospitalization and deaths of the diseases studied with the coverage of Primary Care. Results: Comparing the occurrence of hospitalization and death between 2012 and 2014, a reduction was observed in the Northeast. However, when you correlate with Primary Care coverage, the higher the coverage, the greater the occurrence of injuries. Conclusions: The study revealed the need for reflection and attitudes by managers and health professionals considering the continuity of care for Primary Care users, as well as all financial investments made by the Ministry of Health with the municipalities of northeastern Brazil.

eywords: Primary Health Care; Hypertension; Diabetes Mellitus.

\section{SUMEN}

roducción: Las enfermedades representan la mayoría de las hospitalizaciones y uertes en todo el mundo. Sin embargo, la mayoría de ellos se pueden prevenir diante acciones realizadas en la Atención Primaria. Objetivo: Comparar la bilidad y mortalidad debidas a Enfermedades Crónicas No Transmisibles, 
Hipertensión y Diabetes Mellitus en los años 2012-2014 con la cobertura de Atención Primaria en el noreste de Brasil. Metodología: Se trata de un estudio ecológico y retrospectivo realizado en el noreste de Brasil con datos de 2012 y 2014 con datos disponibles del Departamento de Informática del Sistema Unificado de Salud. Se realizó un análisis descriptivo y analítico que correlacionó las tasas de hospitalización y muertes de las enfermedades estudiadas con la cobertura de la Atención Primaria. Resultados: Al comparar la ocurrencia de hospitalización y muerte entre 2012 y 2014, se observó una reducción en el noreste. Sin embargo, cuando se correlaciona con la cobertura de Atención Primaria, cuanto mayor sea la cobertura, mayor será la ocurrencia de las lesiones. Conclusiones: El estudio reveló la necesidad de reflexión y toma de actitudes por parte de gerentes y profesionales de la salud considerando la continuidad de la atención a los usuarios de Atención Primaria, así como todas las inversiones financieras realizadas por el Ministerio de Salud con los municipios del noreste de Brasil.

Palabras clave: Atención Primaria de Salud; Hipertensión; Diabetes Mellitus. 


\section{Introdução}

As doenças crônicas têm feito parte do maior quantitativo de adoecimento e óbito no mundo, sendo as mais comuns são Hipertensão Arterial Sistêmica (HAS) e Diabetes Mellitus (DM). As complicações ocasionadas por estas comorbidades, acarretam grandes danos à saúde do portador, dificultando a qualidade de vida destes, e grandes gastos ao setor de saúde.

Nesse contexto, o relatório da Organização Mundial de Saúde (OMS) revela que um em cada três adultos com idade entre 25 anos ou mais sofre de Hipertensão Arterial de acordo com relatório divulgado pela OMS. Para Diabetes Mellitus, estimativas recentes indicaram que 11,9 milhões de pessoas entre 20 e 79 anos de idade tinham diabetes no Brasil, o que tornou o país o quarto em número de casos de diabetes no mundo ${ }^{1,2}$.

Essas doenças possuem múltiplos fatores de risco e estão relacionadas com deficiências e incapacidades físicas e funcionais nos sujeitos acometidos e estão inclusas na Lista de Condições Sensíveis à Atenção Primária. Essa lista serve como indicador da atividade em âmbito hospitalar assim como para avaliação da Atenção Primária, sendo essa uma medida indireta da efetividade, do acesso e da qualidade da Atenção Primária à Saúde (APS) ${ }^{3,4}$.

A Atenção Primária é caracterizada e constituída como a porta de entrada preferencial da rede de atenção à saúde, acolhendo usuários e promovendo a vinculação e responsabilização pela atenção a suas necessidades de saúde. $\mathrm{O}$ estabelecimento de mecanismos que assegurem acessibilidade e acolhimento pressupõe uma lógica de organização e funcionamento da $\mathrm{AB}$ adequada às diversas realidades nacionais ${ }^{5,6}$.

Para que os usuários alcancem continuidade do seu tratamento e recebam um iidado em saúde em todos os níveis de atenção, o acesso e o recebimento de um tendimento em saúde que considere a cronicidade do seu adoecimento são condições enciais para a continuidade da atenção necessária. $O$ acesso envolve cinco mensões: disponibilidade dos serviços diante da demanda de usuários, sibilidade, adequação funcional, capacidade financeira e aceitabilidade 7,8. 
Na Atenção Primária estão presentes importantes atributos delimitados como essenciais, sendo o acesso ao primeiro contato, a longitudinalidade do cuidado, a integralidade, a coordenação; e atributos derivados que são a focalização na família, orientação comunitária e competência cultural. Com isso podendo desempenhar as funções de resolubilidade, comunicação e responsabilização ${ }^{9}$.

Considerando a necessidade de atenção aos indicadores de morbidade e mortalidade por DCNT em diferentes regiões do Brasil, dentre elas o Nordeste, o estudo objetivou comparar a morbidade e mortalidade por Doenças Crônicas Não Transmissíveis, Hipertensão Arterial e Diabetes Mellitus nos anos 2012-2014 com a cobertura da Atenção Primária na região Nordeste do Brasil.

\section{Metodologia}

Trata-se de estudo ecológico, retrospectivo, realizado no nordeste brasileiro. A região escolhida é formada por nove estados, sendo eles: Alagoas (AL), Ceará (CE), Bahia (BA), Maranhão (MA), Paraíba (PB), Pernambuco (PE), Piauí (PI), Rio Grande do Norte (RN) e Sergipe (SE).

O período estudado, foram os anos de 2012 e 2014. A escolha do período se deu pela inserção de um programa pelo Ministério da Saúde com objetivo de melhorar a qualidade da assistência e consequentemente os indicadores de morbimortalidade.

Os dados do presente estudo foram coletados da base de dados do Ministério da Saúde. Especificamente, do Departamento de Informática do Sistema Único de Saúde (DATASUS), onde foram coletados os dados de internação (Sistema de Internação hospitalar), de mortalidade (Sistema de Informação sobre Mortalidade) e a cobertura da Atenção Primária (Departamento de Atenção Básica) ${ }^{10}$.

As variáveis dependentes utilizadas foram as taxas de morbidade e mortalidade HAS e DM dos anos de 2012 e 2014. Estas foram calculadas através do número de itos e internados dos portadores de doenças hipertensivas e de diabetes mellitus idido pela população residente multiplicado por 10.000 habitantes. Para os dados população residente foi utilizado a população do censo realizado pelo Instituto 
Brasileiro de Geografia e Estatística (IBGE) no ano de 2010 11. Como variável independente utilizou-se a cobertura da Atenção Primária a qual é expressa em percentual.

Os dados coletados, foram armazenados no Microsoft Excel e em seguida submetidos ao software Statistical Package for the Social Sciences (SPSS), versão 22, onde foram calculadas as taxas e realizada a análise estatística, com cálculos de média, desvio padrão, comparação de média com test $t$ e correlação entre as variáveis dependentes com a variável independente.

O estudo não necessitou de submissão ao Comitê de Ética e Pesquisa, por se tratar de utilização de base de dados secundários, conforme rege a Resolução no510/2016 do Conselho Nacional de Saúde ${ }^{12}$.

\section{Resultados}

A tabela 1 apresenta a distribuição das taxas de mortalidade e morbidade por Hipertensão Arterial Sistêmica (HAS) e Diabetes Mellitus (DM) na Região Nordeste do Brasil nos anos de 2012 e 2014.

Tabela 1: Distribuição das taxas de morbidade e mortalidade por Hipertensão Arterial Sistêmica e Diabetes Mellitus em 2012 e 2014 no nordeste do Brasil. Natal-RN, 2018.

\begin{tabular}{|c|c|c|c|c|c|c|c|}
\hline & \multirow{2}{*}{$\mathbf{N}$} & \multirow{2}{*}{ Média } & \multirow{2}{*}{ Mediana } & \multirow{2}{*}{ DP } & \multicolumn{2}{|c|}{ IC } & \multirow{2}{*}{$\mathbf{P}$} \\
\hline & & & & & Mínimo & Máximo & \\
\hline \multicolumn{8}{|c|}{ Taxa de mortalidade por Hipertensão Arterial Sistêmica } \\
\hline 2012 & 1794 & 3,09 & 2,68 & 2,41 & 2,97 & 3,20 & $<0,01$ \\
\hline 2014 & 1794 & 3,15 & 2,78 & 2,37 & 3,04 & 3,26 & $<0,01$ \\
\hline \multicolumn{8}{|c|}{ Taxa de morbidade por Hipertensão Arterial Sistêmica } \\
\hline 2012 & 1794 & 11,09 & 4,68 & 16,93 & 10,31 & 11,88 & $<0,01$ \\
\hline 2014 & 1794 & 10,29 & 3,84 & 18,46 & 09,44 & 11,15 & $<0,01$ \\
\hline
\end{tabular}




\begin{tabular}{lrrrrrrr}
\hline 2012 & 1794 & 3,63 & 2,54 & 3,25 & 3,51 & 3,75 & $<0,01$ \\
2014 & 1794 & 3,75 & 3,43 & 2,52 & 3,64 & 3,87 & $<0,01$
\end{tabular}

Taxa de morbidade por Diabetes Mellitus

$\begin{array}{rrrrrrrr}2012 & 1794 & 9,52 & 7,03 & 9,55 & 9,08 & 9,97 & <0,01 \\ \mathbf{2 0 1 4} & 1794 & 10,15 & 6,98 & 10,46 & 9,67 & 10,64 & <0,01\end{array}$

Fonte: DATASUS/BRASIL

Na tabela 1, 1794 (100\%) compuseram os municípios da Região Nordeste do Brasil. Observa-se que a média da taxa de mortalidade por Hipertensão Arterial Sistêmica (HAS) teve leve elevação comparando os dois anos. O contrário ocorreu com a média da taxa de internação que diminuiu de 2012 para 2014.

A mortalidade por Diabetes Mellitus (DM) apresentou média mais elevada em relação às médias de HAS, apresentando elevação quando comparados os anos de 2012 e 2014. Porém, ao que se refere à internação por DM, as médias das taxas foram menores quando comparadas com as médias de HAS. Também expressou diminuição nos valores do Desvio Padrão para esses dois anos.

A figura 1 apresenta a correlação entre a taxa de mortalidade por Hipertensão Arterial Sistêmica (HAS) nos anos de 2012 e 2014 e a cobertura de Atenção Primária.

Figura 1 - Comparação entre a taxa de mortalidade por Hipertensão Arterial Sistêmica nos anos de 2012 e 2014 e a cobertura de Atenção Primária. Natal-RN, 2018.

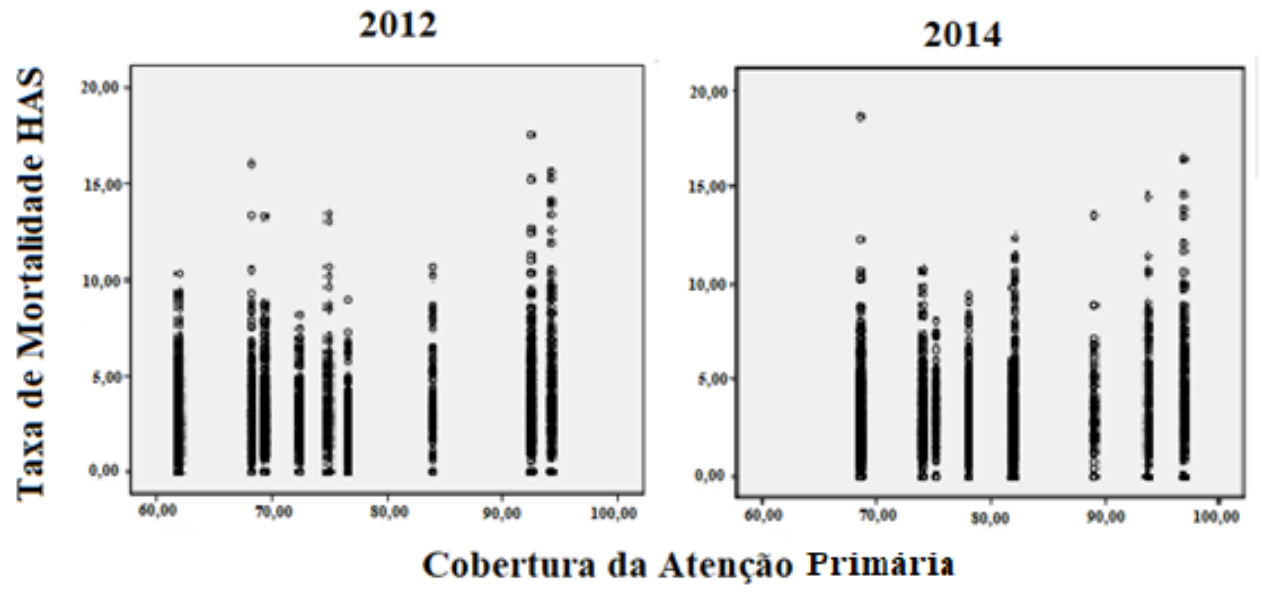

Fonte: DATASUS/BRASIL 


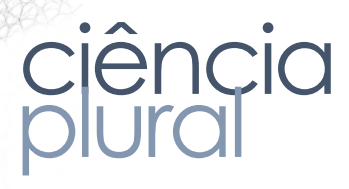

Em relação ao ano de 2012, a cobertura de Atenção Primária mostrou-se em torno de 63\%. Apresentaram Estados do Nordeste com alta cobertura de Atenção Primária e presença de concentração de taxas de mortalidade em torno de 10 óbitos por 10 mil habitantes. Para o ano de 2014, a cobertura de Atenção Primária apresentouse em torno de $69 \%$.

A figura 2 apresenta a correlação entre a taxa de Internação por Hipertensão Arterial Sistêmica (HAS) nos anos de 2012 e 2014 e a cobertura de Atenção Primária.

Figura 2- Comparação entre a taxa de Internação por Hipertensão Arterial Sistêmica nos anos de 2012 e 2014 e a cobertura de Atenção Primária. Natal-RN, 2018.
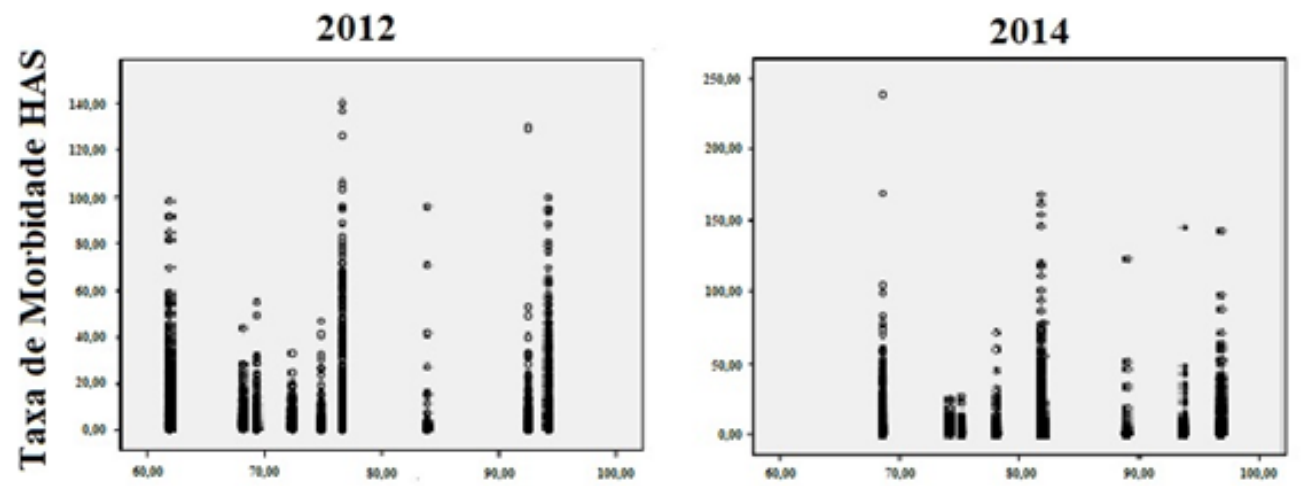

\section{Cobertura da Atenção Primária}

Fonte: DATASUS/BRASIL

A partir da figura 2, pode-se perceber que no ano de 2012 há um número importante de casos de internação por HAS em locais que possuem cobertura de Atenção Primária entre $70 \%$ a $80 \%$. A faixa de locais com cobertura entre $80 \%$ e 90\% apresenta a menor concentração de taxas. Para 2014, as maiores concentrações das taxas de internação estiveram em locais com cobertura em torno de $69 \%$ e $82 \%$.

A figura 3 apresenta a correlação entre a taxa de mortalidade por Diabetes Mellitus nos anos de 2012 e 2014 e a cobertura de Atenção Primária. 
Figura 3- Comparação entre a taxa de mortalidade nos anos de 2012 e 2014 por Diabetes Mellitus e a cobertura de Atenção Primária. Natal-RN, 2018.
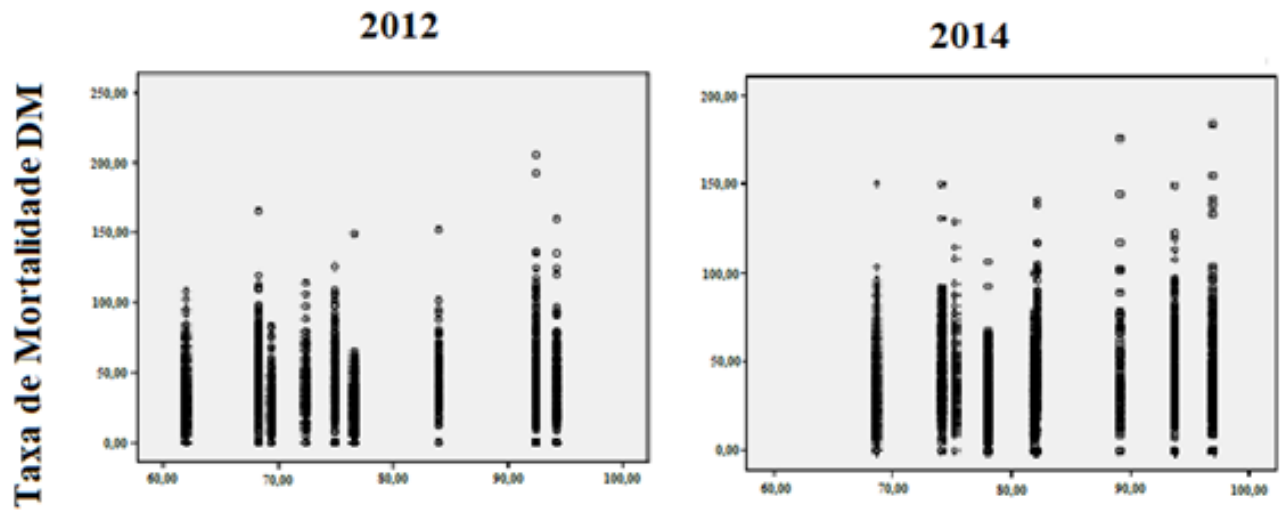

\section{Cobertura da Atenção Primária}

Fonte: DATASUS/BRASIL

No ano de 2012, as maiores concentrações das taxas de mortalidade por Diabetes Mellitus estiveram em locais com cobertura abaixo de $80 \%$ e entre $90 \%$ e $95 \%$. Alguns dados de taxas mostraram-se discrepantes entre 15 e 20 casos por 10 mil.

No ano de 2014, a menor concentração das taxas ocorreu em locais com cobertura de Atenção Primária em torno de 90\%. Mostrou presença de considerável número de casos em locais com alta cobertura entre 90\% e 100\%.

A figura 4 apresenta a correlação entre a internação por Diabetes Mellitus nos anos de 2012 e 2014 e a cobertura de Atenção Primária.

Figura 4- Comparação entre a taxa de internação nos anos de 2012 e 2014 por Diabetes Mellitus e a cobertura de Atenção Primária. Natal-RN, 2018.
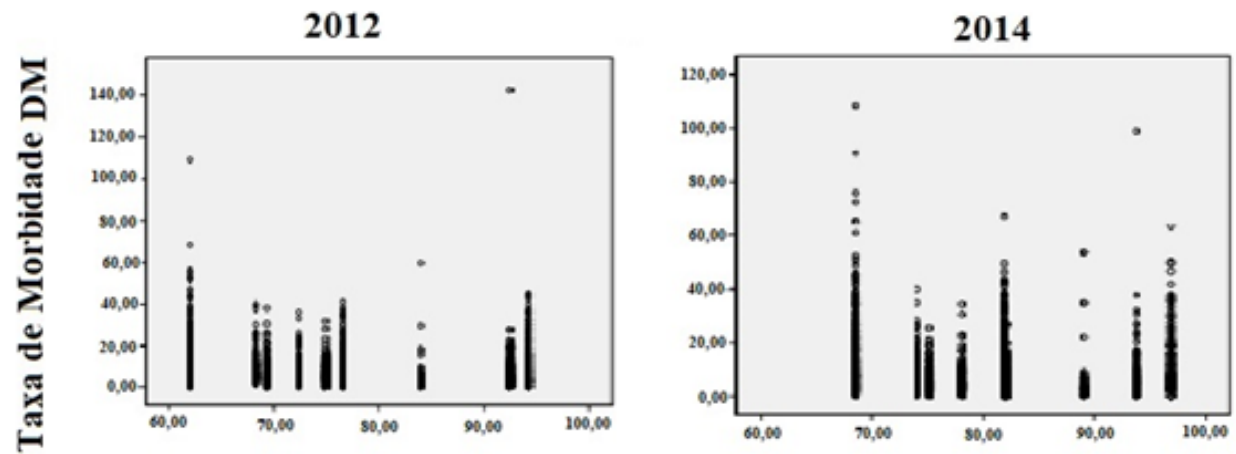

Cobertura da Atenção Primária

Fonte: DATASUS/BRASIL 
No ano de 2012, a menor concentração das taxas ocorreu em locais com cobertura de Atenção Primária em torno de 85\%. Mostrou presença de considerável número de casos em locais com alta cobertura entre $90 \%$ e 100\%. E a maior concentração esteve em locais com cobertura em torno de $63 \%$.

O ano de 2014 mostrou semelhança sobre a concentração das taxas de internações e as de mortalidade por DM, com a menor concentração em locais com cobertura próxima a $90 \%$.

\section{Discussão}

Os dados mostram que, mesmo em locais com alta cobertura de Atenção Primária na região Nordeste, percebe-se a presença de altas concentrações de taxas de mortalidade e internação por Hipertensão Arterial Sistêmica e Diabetes Mellitus.

A discussão para os resultados apresentados é norteada a partir do Modelo de Atenção às Condições Crônicas (MACC) apresentado, no Brasil, por Mendes em 2011. Outra vertente de discussão para esses achados refere-se ao contexto de cobertura da Atenção Primária no Brasil.

Vale ressaltar a existência de evidências de que a atuação adequada dos profissionais e gestores de saúde no nível da Atenção Primária pode colaborar para a redução das hospitalizações e óbitos das DCNT, sobretudo, pela prevenção de complicações micro e macrovasculares na atenção dispensada pelas equipes da Estratégia Saúde da Família ${ }^{13}$.

Contudo, salienta-se que a atuação da Atenção Primária à Saúde no sentido de maximizar sua contribuição para a prevenção e controle de doenças crônicas, requer muito mais que simplesmente um aumento na quantidade de acessos quando se apresenta uma considerável cobertura de serviços ${ }^{14}$.

Macinko ${ }^{14}$ aborda que a análise das hospitalizações decorrentes de Diabetes ellitus e Hipertensão, consiste em uma das formas de monitorar a efetividade das es na Atenção Primária à Saúde, capaz de refletir-se em uma melhor adesão ao tamento, adoção e cultivo de hábitos de vida saudáveis, de maneira a reduzir a 


\section{ciência
piural}

necessidade de hospitalização. A efetividade de ações e o bom funcionamento do sistema de saúde tem como alicerce a qualificação das ações desenvolvidas na APS.

Nesse sentido, as ações de promoção da saúde são estratégias que buscam propiciar qualidade de vida e saúde, por meio do enfrentamento e resolução dos problemas de saúde e seus determinantes. Consiste em um processo de capacitação da comunidade, estimulando-a para a participação no processo de promoção da sua saúde. Não pode ser entendida como prevenção de doenças. O antigo paradigma da saúde, cuja visão voltada para a prevenção, ainda predomina é assimilada por alguns profissionais que apontam dificuldades para desenvolverem atividades com foco na promoção da saúde, sendo essas relacionadas à organização do serviço, quando os sujeitos sinalizaram a demanda excessiva e o pouco tempo para atendê-la e valorização de processos educativos pela clientela ou profissionais 15 .

Destaca-se, no Brasil, desde as últimas décadas, iniciativas do Ministério da Saúde pela institucionalização da avaliação da Atenção Primária e iniciativas de indução para a melhoria, acesso e qualidade nesse nível de atenção. Entre as mais recentes, encontra-se o Programa para Melhoria do Acesso e da Qualidade da Atenção Básica (PMAQ-AB), implantado em 2011, no âmbito da Política Nacional de Atenção Básica (PNAB). O primeiro ciclo do PMAQ-AB, cumprido em 2012, registrou a adesão de $70 \%$ dos municípios brasileiros e incluiu a realização de um censo de estrutura e organização dos serviços de $\mathrm{AB}$ em todo o território nacional. Encontram-se delimitados nesse programa dimensões e indicadores relativos à atenção e assistência para as Doenças Crônicas Não Transmissíveis 16,17.

Aliado às iniciativas de melhoria dos serviços, há que abordar para a problemática de disseminação das DCNT o quanto é necessário atuar sobre os condicionantes e determinantes de saúde, articulando ações mais efetivas do Estado com o propósito de minimizar as desigualdades e coordenar ações intra e intersetoriais, operando um cuidado integral dessas DCNT e seus fatores de risco ${ }^{18}$.

Estudo realizado por Quevedo ${ }^{18}$ aponta que em análise dos planos de saúde dos s de 2012 a 2015, no Brasil, dos 09 Estados da região Nordeste, o tema dos terminantes e condicionantes de saúde foi contemplado apenas em 5 Estados, sendo 
o RN destaque inclusive para outros Estados do Brasil como o Estado que mais teve abordagem sobre o tema. Há Estados que informam que o plano foi elaborado mediante uma análise situacional da população, determinantes e condicionantes da saúde, porém não apresentam no texto.

A pesquisa de Andrade et al. ${ }^{19}$ refere que apesar de o sistema de saúde brasileiro ter sido formulado para garantir acesso universal e igualitário para toda a população, há que considerar em seu desenho institucional misto, que parcela significativa da assistência à saúde é da saúde suplementar e essa realidade parece alimentar a desigualdade no acesso e na utilização de serviços de saúde.

Há que considerar a expansão expressiva da Estratégia Saúde da Família em todo o país sob indução do Ministério da Saúde, atingindo, em junho de 2004, 84\% dos municípios brasileiros, com 20561 equipes e cobertura populacional de 38\% (cerca de 60 milhões de habitantes). Estudo com base no censo do PMAQ-AB no ciclo 1, baseado em dados nacionais censitários, estima e discute as coberturas potenciais de $A B$ no Brasil segundo indicadores selecionados de estrutura. Seus achados apontam para altas coberturas potenciais $(70,0 \% \text { ou superior })^{20}$.

Nesse contexto, correlacionando a necessidade de cobertura de serviços e a importância de abordagem dos DSS, Mendes $^{21}$ apresenta no modelo MACC a necessidade dos modelos de atenção à saúde precisar ser organizado de modo integrado para atender à transição epidemiológica, bem como ao perfil epidemiológico que é colocado com a magnitude do aumento das DCNT e apresenta o MACC considerando a inter-relação com os Determinantes Sociais da Saúde (DSS) que podem influenciar no contexto de adoecimento e resolutividade das necessidades de saúde da população.

Estudo de Rasella et al.,22 analisou o papel da governança sobre a melhoria da aúde da população. No entanto, apesar de amplamente reconhecida como um lemento necessário, é difícil associar efeitos da governança a desfechos na saúde morbidade ou mortalidade). Os achados indicam que a expansão na cobertura da ratégia de Saúde da Família teve um impacto substancial sobre a mortalidade, e uma governança local sólida é importante para a implementação de serviços mais 
eficazes e para a obtenção de melhores resultados de saúde em termos de redução das taxas de mortalidade por causas passíveis de tratamento.

Malta ${ }^{23}$ em estudo realizado a partir do Plano de enfrentamento para as DCNT, apresentou o declínio das taxas de mortalidade pelas quatro principais DCNT em todas as regiões do Brasil, entre 2000 e 2011. Porém para no ano 2000, a Região Sudeste apresentou as taxas mais elevadas, seguida pela região Sul. Em 2011, as taxas de mortalidade mais elevadas mantiveram-se no Sudeste, logo acompanhadas pelas taxas correspondentes às regiões Nordeste e Sul.

Quanto às DCNT são responsáveis por grande custo econômico, repartido entre o sistema de saúde, a sociedade e as famílias. Essas doenças criam um círculo vicioso com a pobreza, impactando negativamente sobre o desenvolvimento macroeconômico dos países, especialmente daqueles de média e baixa renda ${ }^{24}$.

O impacto econômico do DM é expressivo e a saúde pública sofre impactos diretos, pois as hospitalizações recebem importantes parcelas dos recursos da Saúde Pública, conforme abordado em Estudo realizado na região Nordeste cujo objetivo foi verificar as internações por diabetes custeadas pelo SUS, em um período de três anos 19.

Estudos realizados a partir de taxas padronizadas, na região Nordeste, mostrou que a taxa anual de internação entre mulheres apresentou um crescimento de mais de $250 \%$ entre as faixas etárias de 40 a 49 e 50 a 59 anos, mostrando o maior aumento registrado entre as faixas analisadas. A mesma pesquisa leva a refletir sobre o que pode estar por trás desses resultados, se a cultura ou rotina de vida do homem dificulta a procura pelos serviços de saúde e cause um subregistro de suas informações 25.

O estudo de Rasella et al. ${ }^{22}$ mostrou que a forte governança da saúde é fundamental para a cobertura universal de saúde e investigou se a expansão da ESF reduziu a mortalidade sensível à Atenção Básica. O aumento da cobertura da ESF de para $100 \%$ foi associado a uma redução de 6,8\% nas taxas de mortalidade sensível, comparação com nenhum aumento na cobertura da ESF.

As limitações do presente estudo estão relacionadas com os dados brasileiros mortalidade e internações, tais como erros que possam estar relacionados com o 
diagnóstico, a precisão das Declarações de óbito, as mortes associadas com causas mal definidas e possíveis erros nos bancos de dados secundários do DATASUS.

\section{Conclusões}

A comparação das taxas de mortalidade e morbidade por HAS e DM nos anos de 2012 e 2014, apresentam poucas diferenças quando analisadas entre os dois anos e de acordo com a patologia.

Apresentam concentrações desiguais quando analisada a correlação com a cobertura de Atenção Primária, sendo possível perceber que há locais com altas coberturas de Atenção Primária apresentando altas concentrações dessas taxas. Isso revela a necessita de reflexão e tomada de atitude pelos gestores e profissionais de saúde considerando a continuidade do cuidado ao usuário da Atenção Primária, bem como todos os investimentos financeiros realizados pelo Ministério da Saúde junto aos municípios do Nordeste do Brasil.

Os estudos que abordam as DCNT e sua relação com a cobertura e acesso dos serviços de Atenção Primária abordam também a necessidade de se analisar o contexto dos Determinantes Sociais em Saúde e que outras relações podem influenciar para que somente uma alta cobertura de serviços não seja suficiente para se conseguir bons indicadores na saúde.

A região Nordeste do Brasil possui índices consideráveis de cobertura de Atenção Primária com presença da Estratégia de Saúde da Família, logo há que se considerar quais contextos e implicações nos Estados locais influenciam a correlação inversa entre os indicadores de saúde e a cobertura dos serviços nesse nível de atenção.

\section{eferências}

World Health Organization. World Health Statistics 2011 [Internet]. Geneva, itzerland $\quad$ [cited 2018 Mar 15]. Available from: p:/ / apps.who.int/iris/bitstreᄀam/10665/44844/1/ 9789241564441_eng.pdf. 
2. International Diabetes Federation. IDF Diabetes Atlas. 6th ed. [Internet]. Brussels: IDF; 2013 [cited 2017 Dec 3]. Available from: http://www.idf.org/diabetesatlas

3.Brasil. Ministério da Saúde. A vigilância, o controle e a prevenção das doenças crônicas não-transmissíveis: DCNT no contexto do Sistema Único de Saúde brasileiro. Brasília: Ministério da Saúde; 2005 [cited 2018 Mar 8]. Available from: https://bvsms.saude.gov.br/bvs/publicacoes/DCNT.pdf

4. Brasil. Ministério da Saúde. Portaria no 221, de 17 de Abril de 2008. Lista Brasileira de Internações por Condições Sensíveis à Atenção Primária. Diário Oficial [da] República Federativa do Brasil, Brasília: Ministério da Saúde; 2008. [cited 2018 Mar 8]. Available from: http://bvsms.saude.gov.br/bvs/saudelegis/sas/2008/prt0221_17_04_2008.html

5. Brasil. Ministério da Saúde. Portaria $n^{\circ} 2.488$, de 21 de outubro de 2011. Aprova a Política Nacional de Atenção Básica, estabelecendo a revisão de diretrizes e normas para a organização da Atenção Básica, para a Estratégia Saúde da Família (ESF) e o Programa de Agentes Comunitários de Saúde (PACS). Diário Oficial da União, Brasília: Ministério da Saúde; 2011. [cited 2018 Apr 20]. Available from: http://bvsms.saude.gov.br/bvs/saudelegis/gm/2011/prt2488_21_10_2011.html

6. Brasil. Ministério da Saúde. Portaria n².436, de 21 de setembro de 2017. Aprova a Política Nacional de Atenção Básica, estabelecendo a revisão de diretrizes para a organização da Atenção Básica, no âmbito do Sistema Único de Saúde. Diário Oficial da União; Brasília: Ministério da Saúde; 2017. [cited 2018 Mar 28] Available from: https://bvsms.saude.gov.br/bvs/saudelegis/gm/2017/prt2436_22_09_2017.html

7. Assis MMA, Jesus WLA. Access to health services: approaches, concepts, policies and analysis model. Ciênc. Saúde coletiva [Internet]. 2012 [cited 2017 May 10];17(11):2865-875. Available from: http://www.scielo.br/scielo.php?script=sci_ar ttext\&pid=S1413-81232012001100002\&lng=en

8. Figueiredo SV, Custódio LL, Silva AML, Oliveira SR, Figueiredo JV, Gomes ILV. Experiências de acesso à Atenção primária de crianças e adolescentes com deficiências. Rev enferm UFPE on line., Recife, 2017;11(Supl. 12):5197-206.

9. Starfield B. Atenção primária: equilíbrio entre necessidades de saúde, serviços e tecnologia. Brasília, DF: Unesco, Ministério da Saúde, 2002.

10. Departamento de Informática do Sistema Único de Saúde. TabNet. Brasil, 2017.

Instituto Brasileiro de Geografia e Estatística. IBGE. Censo 2010.

Brasil. Resolução $n^{0}$ 510, de 07 de abril de 2016. Procedimentos Metodológicos racterísticos das Áreas de Ciências Humanas e Sociais. Brasília, 2016. 
13. Alfradique ME, Bonolo PF, Dourado I, Lima-Costa MF, Macinko J, Mendonça CS, et al. Internações por condições sensíveis à atenção primária: a construção da lista brasileira como ferramenta para medir o desempenho do sistema de saúde (Projeto ICSAP - Brasil). Cad Saúde Pública 2009; 25(6):1337-1349.

14. Macinko J, Oliveira VB, Turci MA, Guanais FC, Bonolo PF, Lima-Costa MF. The influence of primary care and hospital supply on ambulatory care-sensitive hospitalizations among adults in Brazil, 1999-2007. Am J Public Health. 2011 Oct;101(10):1963-70.

15. Lopes MSV, Machado MFAS, Barroso LMM, Macêdo EMT, Costa RP, Furtado LCS. Promoção da saúde na percepção de profissionais da estratégia saúde da família. Rev Rene. 2013; 14(1):60-70.

16. Brasil. Ministério da Saúde. Autoavaliação para a Melhoria do Acesso e da Qualidade da Atenção Básica: AMAQ. Brasília, 2012. [cited 2017 May 10]. Available from:

http://bvsms.saude.gov.br/bvs/publicacoes/autoavaliacao_melhoria_acesso_quali dade_amaq_2ed.pdf

17. Brasil. Ministério da Saúde. Programa Nacional de Melhoria do Acesso e da Qualidade da Atenção Básica (PMAQ) Manual Instrutivo $3^{\circ}$ Ciclo (2015 - 2016), Brasília, 2015. [cited 2017 May 10]. Available from: http://189.28.128.100/dab/docs/portaldab/documentos/Manual_Instrutivo_3_Cicl o_PMAQ.pdf

18. Quevedo ALA, Bagatini CLT, Bellini MIB, Machado RZ, Guaranha C. Determinantes e condicionantes sociais: formas de utilização nos planos nacional e estaduais de saúde. Trabalho, Educação e Saúde, 2017;15(3), 823-842.

19. Andrade F. Estimating diabetes and diabetes-free life expectancy in Mexico and seven major cities in Latin America and the Caribbean. Rev Panam Salud Publica. 2009 Jul;26(1):9-16.

20. Pocas KC, Freitas LRS, Duarte EC. Censo de estrutura da Atenção Primária à Saúde no Brasil (2012): estimativas de coberturas potenciais. Epidemiol. Serv. Saúde, 2017;26(2): 275-284.

21. Mendes E. O cuidado das condições crônicas na atenção primária à saúde: o imperativo da consolidação da estratégia da saúde da família. Brasília, 2012. [cited 2017 May 10]. Available from: http://bvsms.saude.gov.br/bvs/publicacoes/cuidado_condicoes_atencao_primaria saude.pdf

Hone T, Rasella D, Barreto ML, Atun R, Majeed A, Millett C. Large Reductions In enable Mortality Associated With Brazil's Primary Care Expansion and Strong th governance. Health AFF (Milwood). 2017;36(1): 149-158. 
23. Malta DC, Moura L, Prado RR, Schmidt MI, Duncan BB. Mortalidade por doenças crônicas não transmissíveis no Brasil e suas regiões, 2000 a 2011. Epidemiol. Serv. Saúde [online]. 2014;23(4):599-608.

24. Abegunde DO, Mathers CD, Adam T, Ortegon M, Strong K. The burden and costs of chronic diseases in low-income and middle-income countries. Lancet. 2007;370(9603):1929-1938.

25. Santos FAL, Lima WP, Santos AL, Teston EF, Marcon SS. Hospitalizações por diabetes em adultos e idosos no Ceará, 2001-2012. Epidemiol. Serv. Saúde [Internet]. 2014 Dec [cited 2020 July 30]; 23(4): 655-663. Available from: http:/ / www.scielo.br/scielo.php?script=sci_arttext\&pid=S2237-

96222014000400655\&lng=en 